ORIGINAL

\title{
COMPORTAMIENTO DE LA VARIEDAD Nu OPAL (Gossypium hirsutum L.) BAJO DIFERENTES ARREGLOS ESPACIALES
}

\section{BEHAVIOR OF Nu Opal COTTON VARIETY IN DIFFERENT SPATIAL ARRANGEMENTS}

\author{
Carlos M. Sierra ${ }^{1}$, Luis A. Galvis², Anibal Trebilcok³ ${ }^{3}$ Jorge Cadena ${ }^{4}$ \\ Recibido para evaluación: Mayo 27 de 2010 - Aceptado para publicación: Octubre 11 de 2010
}

\begin{abstract}
RESUMEN
Esta investigación se realizó con el objetivo de determinar el arreglo espacial con mayor potencial de rendimiento de campo de la variedad de algodón Nu Opal bajo las condiciones agroecológicas del Valle del Sinú Medio. Se evaluaron seis densidades de población a partir de la variación de las distancias entre surcos (desde 1,0; 0,$9 ; 0,8 ; 0,7 ; 0,6$ y 0,5 m) y fijando dos plantas por metro. Estas fueron comparadas con la densidad de población utilizada por los agricultores (62.500 plantas ha-1). Los tratamientos fueron dispuestos en un diseño de bloques completos al azar (DBCA) con cuatro repeticiones. La siembra experimental se realizó en el centro experimental CORPOICA CI Turipaná en el segundo semestre del año 2006. Los resultados resaltan que los arreglos espaciales utilizados no afectaron la altura de planta, floración ni los componentes de calidad de la fibra. En cambio, se encontraron diferencias significativas $(P<0,05)$ para el número de botones y el rendimiento de campo. El mayor rendimiento de algodón-semilla y fibra fue mostrado por el tratamiento testigo con 5.370,7 kg ha-1 y 2.028,3 kg ha-1, respectivamente. Cabe destacar que el tratamiento de 40.000 plantas.ha ${ }^{-1}$ presentó rendimientos similares con $5.137,4 \mathrm{~kg} \mathrm{ha}^{-1}$ de algodón-semilla y $1.986,7 \mathrm{~kg} \mathrm{ha}^{-1}$ de fibra, habiendo diferencia numérica pero no estadística $(P>0,05)$ entre ellos.
\end{abstract}

Palabras Clave: Gossypium hirsutum L., densidad de población, evaluación agronómica, rendimiento.

\begin{abstract}
This research was carried out to determine the spatial arrangement with higher yield potential field of Nu Opal cotton variety under the agroecological conditions of the middle Sinu Valley. Were evaluated six different population densities from the variation of the distances among rows (from 1,$0 ; 0,9 ; 0,8 ; 0,7 ; 0,6$ to $0,5 \mathrm{~m}$ ) and setting two plants per meter. These were compared with the density of population used by farmers in the producing area (62.500 plants ha- $\left.{ }^{-1}\right)$. Treatments were arranged in a design of a randomized complete block (RCBD) with four replications. Planting was carried out in the experimental station Turipaná CORPOICA CI in the second half of 2006. The results showed that the spatial arrangements used did not affect neither the expression of plant height and flowering, nor the components of fiber quality. In contrast, significant

\footnotetext{
Ingeniero Agrónomo; Estudiante de Maestría en Ciencias Agronómicas, Universidad de Córdoba, Montería, Colombia. Email: cmariosierra@hotmail.com

${ }^{2}$ Ingeniero Agrónomo; Proexa S.A.

${ }^{3}$ Ingeniero Agrónomo; Esp. Profesor, Facultad de Ciencias Agronómicas, Universidad de Córdoba, Montería, Colombia.

${ }^{4}$ Ingeniero Agrónomo; PhD. Corpoica, Montería, Colombia.
} 
differences $(\mathrm{P}<0,05)$ for the number of buttons and field performance were encountered. The highest yield of cotton-seed and fiber was shown for the control treatment to 5370,7 and 2028,3 kg ha-1 respectively. Notably, the treatment of 40,000 plants ha-1 had similar yields to the control with $5137,4 \mathrm{~kg} \mathrm{ha}^{-1}$ of cotton-seed and $1986,7 \mathrm{~kg} \mathrm{ha}^{-1}$ of fiber, having numerical but not statistical difference $(\mathrm{P}>0,05)$ between them.

Key words: Gossypium hirsutum L., spatial arrangement, density of population, agronomic evaluation, yield.

\section{INTRODUCCIÓN}

En Córdoba la rentabilidad del cultivo de algodón se ha visto disminuida en razón a varios factores como el estancamiento de los rendimientos, la disminución en el área sembrada y los altos costos de producción; incidiendo el valor de la semilla como variable preponderante y fundamental sobre todo en la agricultura de minifundio que se practica en el departamento (Martínez 2005).

Colombia cerró el año 2009 con un área de 35.700 ha sembradas con semillas genéticamente modificadas (transgénicas - GM) en diez departamentos del país, de las cuales 18.784 ha pertenecieron al cultivo de algodón. En 2010 se sembraron 37.657 hectáreas de algodón GM, lo que representó un aumento de más de 18 mil hectáreas respecto del año anterior (Agro-Bio 2010). Durante la temporada 2007/08, en las microrregiones del Valle del Sinú y las Sabanas Colinadas de Sucre y Bolívar, las variedades foráneas ocuparon cerca del 60\% del área sembrada (FINAGRO 2009). En Córdoba, departamento que representó el $71,4 \%$ de las áreas sembradas a nivel nacional, el $29 \%$ de las variedades utilizadas fueron del tipo Nu Opal (CONALGODON 2008). Esto muestra una clara referencia de la tendencia hacia la adopción de genotipos transgénicos por parte de los productores de las subregiones del Caribe húmedo y seco colombiano.
La siembra de un genotipo debe entenderse como una tecnología que requiere del acompañamiento mancomunado de labores para que se exprese su máximo potencial productivo de campo (Cadena 2006). Por esta razón la utilización de un material de tipo transgénico sugiere encontrar estrategias que permitan contrarrestar los elevados costos de producción, máximo cuando esta tecnología no está desarrollada para elevar la productividad de campo y su valor comercial está dado por la venta unitaria de semilla física (FINAGRO 2009).

Teniendo en cuenta que los rendimientos del algodón son afectados por varias prácticas agronómicas, entre estas las densidades de población y los niveles defertilización utilizados (Méndez-Natera 2001), deben determinarse las densidades de población que requiere cada genotipo para hacer más eficiente los procesos productivos y obtener el máximo rendimiento de campo. Cabe destacar que los modelos de siembra convencional incluyen densidades de población relativamente altas, lo que se ha venido aplicando en el país y el Valle del Sinú, el cual sustenta sus producciones en gran parte gracias a una oferta medio ambiental (suelo y clima) ventajosa.

El presente trabajo tuvo como objetivo evaluar diferentes arreglos espaciales con el fin de obtener los máximos rendimientos de campo 
de la variedad transgénica de algodón $\mathrm{Nu}$ Opal, bajo las condiciones del Valle Medio del Sinú.

\section{MATERIALES Y MÉTODOS}

Esta investigación se realizó durante el segundo semestre de la temporada algodonera 2006-2007 en el CI Turipaná de CORPOICA, localizado a $8^{\circ} 5^{\prime}$ de latitud Norte y $75^{\circ} 49^{\prime}$ de longitud Oeste con una elevación de 20 m.s.n.m. En la zona predomina un régimen de precipitaciones con un promedio anual de $1.300 \mathrm{~mm}$, una temperatura promedio de $28^{\circ}$ C y 5,42 horas diarias de brillo solar (IGAC 1996).

Se evaluaron seis arreglos espaciales a partir de la variación de las distancias entre surcos 1,0; 0,$9 ; 0,8 ; 0,7 ; 0,6$ y $0,5 \mathrm{~m}$, fijando dos plantas por metro lineal de surco con una disposición de éstas en forma de triángulo, generándose densidades de población que variaron desde las 20.000 a 40.000 plantas ha-1 (Tabla 1). Estas densidades poblacionales fueron confrontadas a la utilizada por los productores en la zona (62.500 plantas ha-1), la cual tiene una disposición convencional de las plantas en forma de cuadrado $(0,8 \mathrm{~m}$ entre surcos $y$
0,20 m entre plantas). La siembra experimental se Ilevó a cabo mediante el sistema manual tecnificado.

Los tratamientos fueron dispuestos en un diseño experimental de bloques completos al azar (DBCA), con cuatro repeticiones, para un total de 28 unidades experimentales (UE). Cada UE consistió de diez surcos de 10 m de largo, mientras que el tamaño de parcela varió conforme al arreglo espacial de cada tratamiento.

Se midieron variables que describen el comportamiento agronómico de la variedad de algodón Nu Opal en la zona productora, tales como altura de planta (ALPLAN), número de botones florales (BOTON) y floración (FLORA). Así como también los componentes del rendimiento efectivo de campo como rendimiento de Algodón semilla (RENDAS), peso por mota (PEMOT), motas por planta (MOTPLA), rendimiento de fibra (RENFI). Además, se tuvieron en cuenta los componentes de calidad de la fibra como uniformidad (UNIF), elongación (ELONG), resistencia (RESIS), longitud (LONGI) y micronaire (MICRO). Las variables de calidad de fibra fueron medidas luego de realizarse la cosecha del experimento

Tabla 1. Distancias de siembra, Distancia entre plantas, Densidad de población, metros lineales cosechables

\begin{tabular}{ccccc}
\hline Tratamiento & $\begin{array}{c}\text { Distancia entre } \\
\text { surcos }(\mathbf{m})\end{array}$ & $\begin{array}{c}\text { Distancia entre } \\
\text { plantas }(\mathbf{m})\end{array}$ & $\begin{array}{c}\text { Densidad de } \\
\text { población }(\mathbf{h a})\end{array}$ & $\begin{array}{c}\text { Metros cosechables } \\
\left(\mathbf{m} \mathbf{~ h a}^{-1}\right)\end{array}$ \\
\hline Testigo & 0,80 & 0,20 & 62.500 & 12.500 \\
T1 & 1,00 & 0,50 & 20.000 & 10.000 \\
T2 & 0,90 & 0,50 & 22.222 & 11.111 \\
T3 & 0,80 & 0,50 & 25.000 & 12.500 \\
T4 & 0,70 & 0,50 & 28.571 & 14.285 \\
T5 & 0,60 & 0,50 & 33.333 & 16.666 \\
T6 & 0,50 & 0,50 & 40.000 & 20.000 \\
\hline
\end{tabular}


teniendo en cuenta una muestra de $60 \mathrm{~g}$ de fibra de cada UE, las cuales fueron analizadas mediante un instrumento HVI Spectrum del laboratorio de fibras de DIAGONAL. La Clasificación del Algodón se llevó a cabo siguiendo los parámetros del Departamento de agricultura de los Estados Unidos EEUU (USDA) (DIAGONAL 2000).

Los datos obtenidos en campo fueron almacenados en sendos libros de anotaciones. Los respectivos análisis de varianza (ANAVA) para cada variable se realizaron con la ayuda del programa SAS v 9.0, utilizando el siguiente modelo estadístico:

$$
Y i j=\mu+T i+B j+\in i j
$$

Donde: $Y_{i j}=$ Efecto del i-ésimo tratamiento en la j-esima repetición; $\mu=$ media general, $T_{i}=$ efecto del i-ésimo tratamiento, $\mathrm{B}=$ efecto de la j-esima repetición, $\varepsilon_{\mathrm{ij}}=$ error aleatorio, con los supuestos usuales $\varepsilon_{\mathrm{ij}} \sim \mathrm{NI}\left(0, \sigma^{2}\right)$.

En aquellas variables en donde el análisis de varianza detectó diferencias significativas, se realizaron pruebas de separación de medias usando la Prueba de Rango múltiple de DUNCAN.

\section{RESULTADOS Y DISCUSION}

El comportamiento de la altura de planta a través de los distintos arreglos espaciales fue muy similar durante el periodo de evaluación comprendido desde los 33 hasta los 96 dde (días después de emergencia). El análisis estadístico no detectó diferencias significativas $(p>0,05)$ por efecto de los tratamientos evaluados, exceptuando los muestreos realizados a los 47, 89 y 96 dde donde el tratamiento de 20.000 plantas ha-1 $(60,6 \mathrm{~cm})$ y los tratamientos de
25.000 y 22.222 plantas ha ${ }^{-1}$ con 125,2 y 131 $\mathrm{cm}$ respectivamente, mostraron los mayores promedios de altura de planta como puede verse en la figura 1. El promedio de altura alcanzado por las plantas en la medición final fue de $123,89 \mathrm{~cm}$.

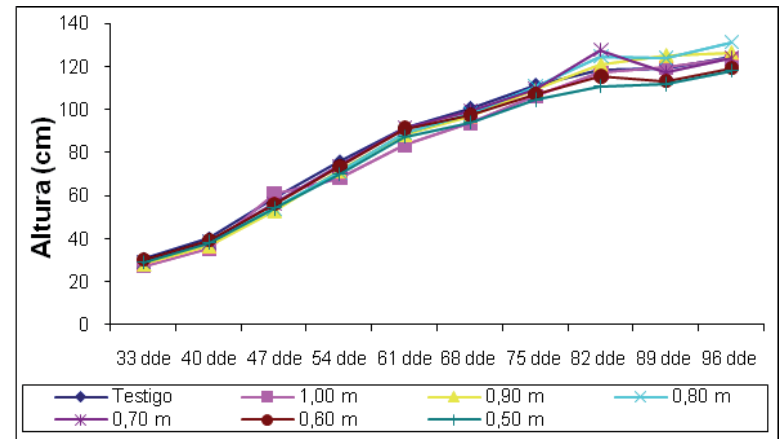

Figura 1. Efecto de siete densidades de población sobre el comportamiento de la altura de planta en la variedad de algodón Nu Opal.

La emisión del botón floral en la planta de algodón de la variedad $\mathrm{Nu}$ Opal en esta investigación alcanzó a los 54 dde la máxima emisión de botones florales, los cuales coinciden con los muestreos realizados en los diferentes tratamientos. A partir de la fecha la emisión de botones disminuye hasta los 96 dde, tal y como lo muestra la figura 2. De esta forma, en el muestreo realizado a los 54 dde se presentan diferencias significativas $(p<0,05)$ y los tratamientos con 28.571 y 25.000 plantas ha $^{-1}$ mostraron la mayor emisión de botones en promedio con 23,65 y 23,5, respectivamente. Este comportamiento se mantuvo hasta los 61 dde donde vuelven a presentar los mayores promedios de emisión de botones $(37,7$ y $35,45)$. Cabe destacar que durante este periodo el tratamiento testigo fue el que mostró los promedios más bajos de emisión de botones con 14,95 y 20,2, respectivamente. 


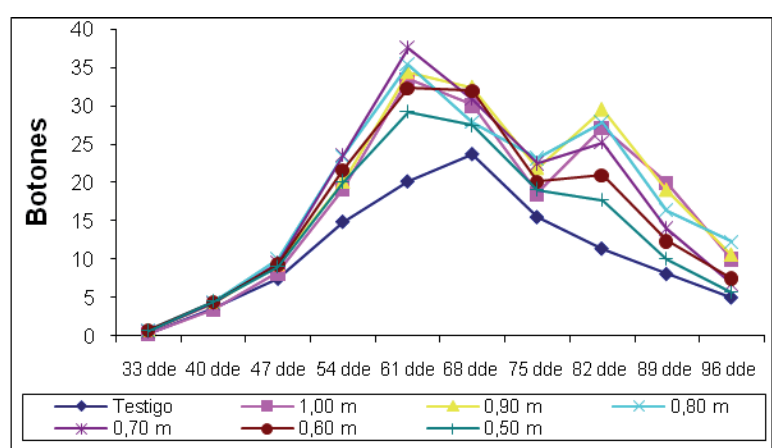

Figura 2. Efecto de siete densidades de población sobre el comportamiento del número de botones en la variedad de algodón $\mathrm{Nu}$ Opal

En el muestreo tomado a los 82 dde, el análisis detectó diferencias altamente significativas entre tratamientos, donde los tratamientos con mayor número de botones emitidos fueron los de 22.222 y 25.000 plantas ha-1 con 29,6 y 27,91 , respectivamente, en tanto que el testigo registró el menor promedio de emisión con 11,45 botones florales.

Para la variable floración, comportamiento similar a la emisión de botones florales se registró en los diferentes tratamientos sometidos a estudio, lo cual guarda relación con lo argumentado para la emisión de botones florales. El mayor número de flores por planta se presentó a los 54 dde, con 2,67 flores. El análisis de varianza no detectó diferencia significativa entre tratamientos. Los tratamientos con 22.222 y 25.000 plantas ha $^{-1}$ promediaron 3 flores por planta, mientras el tratamiento con 20.000 plantas ha-1 solo 2, siendo el promedio más bajo para dicha lectura.

Diferencias significativas entre tratamientos al nivel del $(p<0,01)$ fueron encontradas en los muestreos realizados a los 82 y 89 dde, en la que se presentó el segundo pico de floración.
En los muestreos se observó un promedio de dos (2) flores por planta en los tratamientos con 20.000 plantas y 22.222 plantas ha-1, respectivamente. En el tratamiento testigo la floración fue menor (Figura 3). De forma similar Camargo y Arcón (2007) registraron diferencias estadísticas significativas $(P<0,05)$ a los 94 y 109 dde y altamente significativas $(\mathrm{P}<0,01)$ a los 116 dde, utilizando densidades de población desde 20.000 a 40.000 plantas ha-1 en la variedad Nu Opal.

El rendimiento de campo (RENDAS) arrojó diferencias significativas $(p<0,05)$ entre los tratamientos, donde el mayor valor correspondió al tratamiento testigo con un rendimiento de 5.370,7 $\mathrm{kg} \mathrm{ha}^{-1}$ de algodón - semilla, seguido del tratamiento de 40.000 plantas ha-1 con 5.137,4 kg ha-1, superando al resto de tratamientos en el ensayo (Tabla 2). Los rendimientos más bajos se presentaron en los tratamientos de 22.222 plantas ha $^{-1}$ y 33.333 plantas ha-1 con 4.351,3 kg ha-1 y $4.288,4$ kg ha $^{-1}$ respectivamente. Esta situación también se presentó para el RENFI, donde el análisis de varianza arrojó diferencias significativas entre tratamientos $(p<0,05)$. La mayor producción de fibra se obtuvo también en el tratamiento testigo con 2.028,3 kg fibra ha-1, seguido del

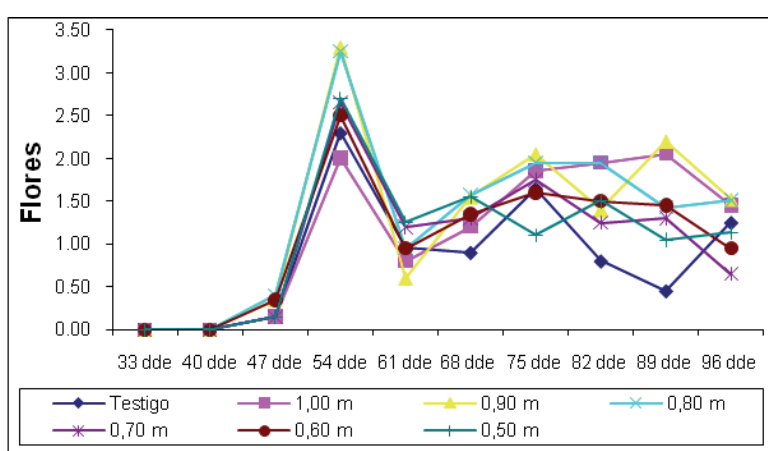

Figura 3. Efecto de siete densidades de población sobre el comportamiento de la floración en la variedad de algodón Nu Opal 
Tabla 2. Efecto de siete densidades de población sobre el comportamiento del Rendimiento de algodónsemilla, Peso de mota, Mota por planta, Uniformidad, Elongación, Resistencia, Longitud y Micronaire de la fibra en la variedad de algodón Nu Opal

\begin{tabular}{|c|c|c|c|c|c|c|c|c|c|}
\hline $\begin{array}{c}\text { PLANTAS } \\
\text { ha }^{-1}\end{array}$ & $\begin{array}{l}\text { RENDAS } \\
\left(\mathrm{Kg} \mathrm{ha}^{-1}\right)\end{array}$ & $\begin{array}{c}\text { RENFI } \\
\left(\mathrm{Kg} \mathrm{ha}^{-1}\right)\end{array}$ & $\begin{array}{c}\text { PEMOT } \\
(\mathrm{g})\end{array}$ & $\begin{array}{l}\text { MOTPLA } \\
\left(\mathbf{N}^{\circ}\right)\end{array}$ & $\begin{array}{l}\text { UNIF } \\
(\%)\end{array}$ & $\begin{array}{c}\text { ELONG } \\
(\%)\end{array}$ & $\begin{array}{c}\text { RESIS } \\
\left(\mathrm{g} \mathrm{tex}^{-1}\right)\end{array}$ & $\begin{array}{l}\text { LONGI } \\
\text { (pulg) }\end{array}$ & $\begin{array}{c}\text { MICRO } \\
\left(\mathrm{mg} \mathrm{pulg}^{-2}\right)\end{array}$ \\
\hline 62.500 & 5371 a & 2028,3 a & 5,54 a & $18,95 \mathrm{c}$ & $84,50 \mathrm{a}$ & 5,18 a & 31,85 a & $1,19 b$ & $4,54 \mathrm{a}$ \\
\hline 20.000 & 4709 abc & $1826,7 \mathrm{ab}$ & 5,59 a & $36,35 \mathrm{a}$ & 83,72 a & 5,30 a & 32,18 a & $1,19 \mathrm{ab}$ & 4,64 a \\
\hline 22.222 & 4351 c & $1719,5 \mathrm{ab}$ & 5,43 a & $36,55 \mathrm{a}$ & 84,78 a & 5,30 a & $32,03 \mathrm{a}$ & $1,20 \mathrm{ab}$ & 4,52 a \\
\hline 25.000 & 4732 abc & $1791,1 \mathrm{ab}$ & 5,67 a & 33,6 a & 84,33 a & 5,33 a & 33,08 a & $1,23 \mathrm{ab}$ & $4,60 \mathrm{a}$ \\
\hline 28.571 & 4647 bc & $1812,7 \mathrm{ab}$ & 5,75 a & $28,45 \mathrm{~b}$ & 85,05 a & 5,28 a & 32,15 a & $1,21 \mathrm{ab}$ & 4,64 a \\
\hline 33.333 & $4288 \mathrm{c}$ & 1636,7 b & 5,56 a & $28,15 b$ & 84,65 a & 5,23 a & $32,25 \mathrm{a}$ & $1,22 \mathrm{ab}$ & 4,46 a \\
\hline 40.000 & $5137 \mathrm{ab}$ & 1986,7 a & 5,43 a & $23,05 \mathrm{c}$ & 85,13 a & 5,23 a & $32,68 \mathrm{a}$ & $1,23 \mathrm{a}$ & $4,47 \mathrm{a}$ \\
\hline Media & 4741.6 & 1828,53 & 1828,53 & 29,3 & 84,59 & 5,26 & 32,31 & 1.212 & 4,56 \\
\hline CV & 8,65 & 9,67 & 9,67 & 11,585 & 1,108 & 2,485 & 3,89 & 1,867 & 5,16 \\
\hline Significancia & $*$ & $*$ & $*$ & $* *$ & ns & ns & $\mathrm{ns}$ & ns & ns \\
\hline
\end{tabular}

tratamiento de 40.000 plantas ha-1 con 1.986,7 kg fibra ha-1.

Este resultado controvierte lo expuesto por Camargo y Arcón (2007), quienes utilizaron densidades de población desde las 20.000 a 40.000 plantas ha $^{-1}$ en la variedad Nu Opal, y no encontraron diferencias estadísticas significativas $(\mathrm{P}>0,05)$ para el rendimiento total por hectárea; esto pudo deberse a que los autores omiten el uso de un tratamiento testigo, el cual en esta investigación hace referencia al sistema de siembra convencional utilizado en la zona por los productores (62.500 plantas ha $\left.{ }^{-1}\right)$. Este fenómeno tendría también explicación si se considera que, el comportamiento de los genotipos difieren en el tiempo (Rodríguez y Rodríguez 1999) y espacio por factores genéticos y la interacción de éstos con el ambiente (factores predecibles e impredecibles), más aún cuando se trata de genotipos foráneos no adaptados completamente a los sistemas de producción de los países tropicales (Cadena 2006). Por tal motivo, es muy probable que los rendimientos de campo difieran de una temporada a otra (Jarma y Mendoza 1999).
La variable PEMOT no mostró diferencias significativas $(\mathrm{P}>0,05)$ entre tratamientos, en tanto que el promedio general fue de 5,57 g (Tabla 2), lo que indica que la expresión de este carácter no se afecta ni presenta diferencias estadísticas al utilizar las densidades de población planteadas en esta investigación. Esto concuerda con lo reportado por Gaytan-Mascorro et al. (2004); Jarma et al. (2002), donde el peso de la mota no arrojó diferencias significativas por la utilización de diferentes densidades de población en surcos ultraestrechos. Por su parte, Camargo y Arcón (2007), registraron diferencias estadísticas significativas $(\mathrm{P}<0,05)$ para peso de mota de tal manera que el mayor y menor peso promedio de mota fue de 6,4 y 4,3 g respectivamente, lo cual no se advierte en esta investigación. Este resultado también difiere de lo argumentado por Fuentes y Monterrosa (2002), quienes además anotan que el peso de la mota disminuyo linealmente con el incremento de la densidad poblacional.

La variable MOTPLA arrojó diferencias altamente significativas entre tratamientos $(p<$ 0.01 ), todos los tratamientos a su vez superaron 
al testigo. Los tratamientos con mayor número de motas expresadas fueron los de 22.22 y 20.000 plantas ha-1 con 36,55 y 36,35 motas por planta respectivamente, mientras que el tratamiento testigo expresó en promedio 18,9 motas en sus plantas. Se pudo observar una mayor concentración de motas por planta a medida que las densidades de población disminuían. Por tanto, existe un claro efecto de las densidades poblacionales utilizadas sobre esta variable (Tabla 2). Esto puede tener explicación si se tiene en cuenta que, a menores densidades poblacionales disminuye la competencia por espacio y nutrientes (Guinn 1982; Rodríguez 2000), a la vez que se desarrollan un mayor número de ramas reproductivas por efecto de la compensación fisiológica existente en la especie. GaytanMascorro et al. (2004) argumentan al respecto que, al incrementar las densidades de población se redujo el número y peso de motas por planta, pero el rendimiento unitario no se afectó ya que la reducción del rendimiento por planta fue compensado por una mayor densidad poblacional. Estos resultados indican que los mayores rendimientos obtenidos por el tratamiento testigo no se deben a una mayor carga de la planta.

En general, los rendimientos de algodónsemilla y fibra obtenidos en esta investigación fueron muy superiores a los promedios de la región, indicando la ventaja de los tratamientos evaluados (Tabla 1).

En cuanto a los componentes de calidad de la fibra, se detectaron diferencias significativas $(\mathrm{P}<0,05)$ sobre la longitud de la fibra. Esto concuerda con lo argumentado por GaytanMascorro et al. (2004) y Méndez-Natera (2006), quienes reportaron diferencias significativas $(\mathrm{P}<0,05)$ para la longitud de la fibra, en tanto que variables como la resistencia, elasticidad y Micronaire no modificaron su comportamiento $(P>0,05)$. En promedio las fibras alcanzaron una longitud de 1.21 pulgadas lo cual es clasificado internacionalmente como "media" (DIAGONAL 2000).

De igual forma no se presentaron diferencias significativas $(P>0,05)$ sobre la resistencia de la fibra. En promedio se obtuvo una resistencia de 32,31 gramos tex-1, lo cual se clasifica como muy resistente. Esto controvierte lo reportado por Palomo et al. (1999), quienes al hacer incrementos en la densidad de población, encontraron que disminuía la resistencia de la fibra.

Las densidades poblacionales evaluadas no afectaron el micronaire de la fibra, ni se detectaron diferencias significativas $(P>0,05)$. Según las tablas internacionales de clasificación del micronaire de la fibra, todos los tratamientos están dentro del rango de clasificación "promedio" los cuales son adecuados para el procesamiento textil. Esto guarda relación con lo argumentado por Camargo y Arcón (2007), donde los tratamientos presentaban micronaire entre 4,3-4,5 mg pulg-2 ${ }^{-2}$ clasificándose como un rango de finura promedio (4 - 4,9 $\mathrm{mg} \mathrm{pulg}^{-2}$ ).

La uniformidad de longitud de la fibra en todos los tratamientos se encuentran en un rango de "muy uniforme" a "excelente", por lo tanto debe esperarse un mayor contenido de fibras de longitud 1,21 pulg en las muestras analizadas. Esta variable tampoco fue afectada por los tratamientos, no arrojando diferencias estadísticamente significativas $(P>0,05)$. 
La elongación de la fibra se mantiene en todos los tratamientos y no varía, por lo que esta no se ve afectada por las densidades poblacionales ensayadas. No hay diferencias significativas entre tratamientos $(P>0,05)$. Anteriormente Camargo y Arcón (2007) encontraron que la elongación de la fibra de la variedad de algodón Nu Opal en distintas densidades de población se clasificó como promedia, lo cual corresponde con lo encontrado en esta investigación.

\section{CONCLUSIONES}

Los arreglos espaciales en el cultivar de algodón $\mathrm{Nu}$ Opal, no influyeron en la altura de plantas y número de botones florales en los diferentes tratamientos considerados en el estudio.

El tratamiento de 40.000 plantas ha-1 ${ }^{-1}$, registró el mayor rendimiento de fibra con $1986 \mathrm{~kg} \mathrm{ha}^{-1}$, lo que constituye una ventaja agronómica si se considera el alto precio de la semilla; ya que se traduce en un ahorro de alrededor del 36\% en el consumo de semilla para la siembra de una hectárea.

Los arreglos espaciales no afectaron la expresión de las variables de calidad de fibra como resistencia, elongación, uniformidad y micronaire.

\section{REFERENCIAS}

\section{Asociación de Biotecnología Vegetal Agrícola} (Agro-Bio). 2010. Transgénicos en el mundo/Región Andina/Colombia. http:// www.agrobio.org/fend/index.php?op= YXA9I2JXbDQmaW09I016UT0=
Cadena, J. 2006. CORPOICA M-123: Nueva variedad de Algodón de fibra media con adaptación al Caribe Húmedo. http:// www.turipana.org.co/Algodon.html [21 Junio 2009].

Camargo, L y Arcón, L. 2007. Evaluación agronómica de diferentes densidades poblacionales en algodón (Gossypium hirsutum I.) variedad NuOpal bajo una distribución espacial organizada en el valle del sinú medio. Tesis Ingeniero Agrónomo. Universidad de Córdoba, Montería.

CONALGODON. 2008. Desempeño del sector algodonero 2005 y perspectivas 2006. Confederación Colombiana de Algodón. http://www.conalgodon.com/ portal/index.php [3 Julio 2009].

DIAGONAL. 2000. La Clasificación del Algodón. Departamento de agricultura de los Estados Unidos EEUU (USDA), Estación Experimental Agropecuaria Sáenz Peña (INTA). http://www.inta.gov. ar/saenzpe/algodon/clasifalgodon.pdf [17 Julio 2009].

FINAGRO. 2009. Sistema de información sectorial. Fondo para el financiamiento del sector agropecuario. http://www. finagro.com.co/html/cache/gallery/GC8/G 11/arroz.pdf [17 Abril 2009]

Fuentes, R. y Monterrosa, J. 2002. Respuesta agronómica de la variedad de algodón DP 5415 sembrado en tres densidades de población evaluados bajo condiciones del medio Sinú. Tesis Ingeniero 
Agrónomo. Universidad de Córdoba, Montería.

\section{Gaytán-Mascorro, A., Palomo A., Reta D.,} Godoy S. y García E. 2004. Respuesta del algodón cv. Cian precoz al espaciamiento entre surcos y densidad poblacional. Revista Phyton 73(1):57-67.

Guinn, G. 1982. Cause of square and boll shedding in cotton. Plant Physiol. 87: 629-631.

\section{Instituto Geográfico Agustín Codazzi (IGAC).} 1996. Diccionario geográfico de Colombia. Tomo 3. Tercera edición, Bogotá, p1858.

Jarma, A., Morales W. y Contreras A., 2002. Surcos ultra angostos en la nueva variedad de algodón corpoica M 123 en el valle del Sinú. $2^{\circ}$ Seminario Nacional algodonero. Memorias: 216, Corpoica, Cereté, p23 - 29.

Jarma, A y Mendoza. 1999. Contribución del mejoramiento genético del algodonero y la productividad en el caribe Colombiano. VI Congreso Sociedad Colombiana de Fitomejoramiento y Producción de Cultivo, memorias: 64, Universidad de los Llanos, VillavicencioMeta, p34 -39.

Martínez H. 2005. La cadena de algodón en Colombia, una mirada global de su estructura y dinámica, 1991-2005. Ministerio de agricultura y desarrollo rural, agrocadenas, Bogotá, Documento de trabajo no. 53 .
Méndez-Natera, J. 2001. Efecto de dos densidades de población y dos niveles de fertilización sobre algunos caracteres vegetativos de tres cultivares de algodón (Gossypium hirsutum L.) en la sabana de Jusepín, Estado Monagas. Revista UDO agrícola 1(1):11-17

Méndez-Natera, J. 2006. Efecto de la densidad de población y la fertilización sobre las características de los frutos de algodón (Gossypium hirsutum L.). Revista Idesia. 24(3):39-47.

Palomo, G.; Gaytán, A. y Godoy, S. 1999. Respuesta de cuatro variedades de algodón (Gossypium hirsutum L.) a la densidad poblacional. Fitotecnia Mexicana 22(1):43-49.

\section{Rodríguez, N y Rodríguez, F. 1999.} Comportamiento de dos genotipos comerciales de algodonero Gossypium hirsutum L. con tres densidades de población sembrados tardíamente en el Sinú medio. Tesis Ingeniero Agrónomo. Universidad de Córdoba, Montería p115.

Rodríguez, L. 2000. Densidad de población vegetal y producción de materia seca. Bogotá: Revista Comalfi 27(1):12-15. 